\title{
Nonstrange quark stars from an NJL model with proper-time regularization
}

\author{
Qingwu Wang $\odot{ }^{1, *}$ Chao Shi ${ }^{2, \dagger}$ and Hong-Shi Zong $\oplus^{3,4, \$}$ \\ ${ }^{1}$ College of Physics, Sichuan University, Chengdu 610064, China \\ ${ }^{2}$ Department of Nuclear Science and Technology, Nanjing University of Aeronautics and Astronautics, \\ Nanjing 210016, China \\ ${ }^{3}$ Department of Physics, Nanjing University, Nanjing 210093, China \\ ${ }^{4}$ Joint Center for Particle, Nuclear Physics and Cosmology, Nanjing 210093, China
}

(Received 18 August 2019; published 3 December 2019)

\begin{abstract}
The structure of a light quark star is studied within a new two-flavor Nambu-Jona-Lasinio model. By retaining the contribution from the vector term in the Fierz-transformed Lagrangian, a two-solar-mass pure quark star is achieved. To overcome the disadvantage of three-momentum truncation in the regularization procedure, we introduce the proper-time regularization. We also employ the newly proposed definition of vacuum pressure [J. Phys. G 45, 105001 (2018)], in which the quasi-Wigner vacuum (corresponding to the quasi-Wigner solution of the gap equation) is used as the reference ground state. The free parameters include only a mixing constant $\alpha$ which weighs the contribution from the Fierz-transformed Lagrangian. We constrain $\alpha$ to be around 0.9 by the observed mass of pulsars PSR J0348 + 0432 and PSR J1614-2230. We find that the calculated surface energy density meets the requirement $\left(>2.80 \times 10^{14} \mathrm{~g} / \mathrm{cm}^{3}\right)[$ Phys. Rev. D 99, 043001 (2019)]. Besides, for a 1.4-solar-mass star, the deformability $\Lambda$ is calculated, which is consistent with a recent analysis on the binary neutron star merger GW170817 with $\Lambda$ in $(0,630)$ for large component spins and $300_{-230}^{+420}$ when restricting the magnitude of the component spins [Phys. Rev. X 9, 011001 (2019)], and satisfies the constraints $200<\Lambda<800$ of early works.
\end{abstract}

DOI: $10.1103 /$ PhysRevD.100.123003

\section{INTRODUCTION}

Investigations of dense matter are an important part of studying strong interactions. The experimental data on the ground tell us that the ground state of the strong interacting baryon at a zero density is a nucleon. At a nonzero density, when the quark chemical potential is higher than the strange quark mass, the strange quark matter may be the ground state [1]. Therefore, the observed pulsars may be quark stars rather than traditional neutron stars. After the discovery of two-solar-mass pulsars [2-5], many theoretical models about a nonstrange star were excluded, because they lead to equations of state (EOSs) that were too soft. Considering the EOS of a strange baryon (hyperon), the maximum pulsar mass is still lower than $2 M_{\odot}$. But the structure of neutron stars can be explored by considering the modified theory of gravity [6,7], introducing a strong

*qw.wang@scu.edu.cn

†shichao0820@gmail.com

zonghs@nju.edu.cn

Published by the American Physical Society under the terms of the Creative Commons Attribution 4.0 International license. Further distribution of this work must maintain attribution to the author(s) and the published article's title, journal citation, and DOI. Funded by SCOAP. electromagnetic field [8-10], or introducing a high-speed rotation effect [11-13] to obtain a $2 M_{\odot}$ neutron star. Recently, one paper indicates that stable hadron matter can be a nonstrange quark state when the baryon number is greater than a certain minimum value [14]. Quark matter with only $u$ - $d$ quarks can be the ground state of baryonic matter other than the $u$ - $d$-s strange quark matter $[1,15,16]$. Therefore, if the observed pulsar is a nonstrange quark star and regardless of all other corrections, a suitable EOS becomes necessary.

Studying the EOS at an extreme environment usually resorts to effective field theories, and the Nambu-JonaLasinio (NJL) model is one of them. With a few parameters fitted to the low-energy experimental data, the NJL model and its many generalizations provided much information on both hadron physics and QCD matter at a finite temperature and density $[17,18]$. We note that, within the NJL model, there are two equivalent descriptions, i.e., the original Lagrangian density $L_{\mathrm{NJL}}$ and its Fierz transformation $L_{\text {Fierz }}$. After doing the mean-field approximation, $\left\langle L_{\mathrm{NJL}}\right\rangle$ contains only the Hartree term, while $\left\langle L_{\text {Fierz }}\right\rangle$ contains only the Fock term. When calculating the quark condensation, the parameters are calibrated to reproduce the physical pion meson mass and decay constant. In this way, the two descriptions are equivalent. However, when considering finite density matter, the effective chemical potentials given 
by the two Lagrangians are different at the mean-fieldapproximation level: The effective potential from the Fierz-transformed Lagrangian has a contribution from quark vector density $\left\langle\psi^{\dagger} \psi\right\rangle$. Actually, without the Fierz transformation, the standard approach of the mean-field approximation is considered to be not self-consistent theoretically [19]. We, therefore, propose a self-consistent treatment that combines the $\left\langle L_{\mathrm{NJL}}\right\rangle$ and $\left\langle L_{\text {Fierz }}\right\rangle$ linearly with a weighting parameter $\alpha$ and study its implication on QCD matter at a finite density [20]. Based on this, our previous study [21,22] has shown that two-flavor quark matter could be more stable than three-flavor quark matter, unlike Witten's prediction based on MIT bag model [1]. As stated in Ref. [20], the parameter $\alpha$ used to reflect the weight of different interaction channels cannot be given in advance by the mean-field theory. It must be determined by related experimental data of high-density strong interacting matter. The neutron star provides such a laboratory. One motivation of this paper is to determine the $\alpha$ through current astronomical observations on neutron stars.

In this paper, we will extend our study in several new aspects. We first employ the proper-time regularization rather than the three-momentum cutoff scheme, since the latter limits the value of $\alpha<0.9$ and, hence, is considered to be less reliable [22]. Note that, for a nonrenormalizable theory, the regularization scheme could play an important role in making physical predictions. Testing with different regularization schemes, therefore, provides a qualitative check for consistency. Second, when calculating the EOS, there is a free parameter, i.e., the bag constant $B$. The bag constant $B$ gives the pressure of quark matter at a zero temperature and zero density. Usually, it is treated as a phenomenological parameter and determined by experimental requirements [21-26]. It has a great influence on the EOS and, consequently, the mass-radius relation of the neutron star. We should be more careful in choosing its value. Experimental requirements [27-29] suggest a typical value about $(120 \mathrm{MeV})^{4}$, but it is also calculable in effective theories. A traditional treatment is to subtract the thermodynamic potential of the current quark from the thermodynamic potential corresponding to the NambuGoldstone solution, i.e., $B=\Omega\left(m_{\text {current }}\right)-\Omega\left(M_{\text {Nambu }}\right)$ [17]. But the current quark is not the solution to the gap equation. We therefore use a recently proposed definition $B=\Omega\left(M_{\text {Wigner }}\right)-\Omega\left(M_{\text {Nambu }}\right)[26,30,31]$, i.e., to subtract from the thermodynamic potential corresponding to the Wigner-Weyl solution. Such a definition is more theoretically self-consistent, since the Wigner-Weyl solution is another (although unphysical) solution to the quark gap equation in the NJL model. The bag constant is now the pressure difference between the Nambu and Wigner phases. It is an intrinsic quantity within the NJL model instead of an input parameter as in Ref. [22]. Finally, we determine the weighting factor $\alpha$ from the experimental data. Besides the observations of the pulsars mass, the tidal deformability measurement from the neutron star merger GW170817 [32-37] has also been used to constrain the stiffness of the EOS. We will check if our predictions agree with other current observations on, e.g., the radius and surface energy of a star.

This paper is organized as follows: In Sec. II, we introduce the NJL model and its Fierz transformation. The weighting factor $\alpha$ is introduced here, and its effects on the quark mass and quark number density are presented. In Sec. III, the EOS, mass-radius relation, and tidal deformability of the stars are calculated. The effect of $\alpha$ on the EOS is presented. In the end, a short summary is given.

\section{THE NJL MODEL AND ITS FIERZ TRANSFORMATION}

The standard NJL model is a model of QCD with fourquark interaction $[17,18]$. Beyond the chiral limit, the twoflavor Lagrangian is

$$
\mathcal{L}_{\mathrm{NJL}}=\bar{\psi}(i \not \partial-m) \psi+G\left[(\bar{\psi} \psi)^{2}+\left(\bar{\psi} i \gamma^{5} \vec{\tau} \psi\right)^{2}\right]+\mu \psi^{\dagger} \psi .
$$

In the mean-field approximation,

$$
\mathcal{L}_{1}=\bar{\psi}(i \not \partial-m) \psi+2 G \sigma_{1} \bar{\psi} \psi+\mu \psi \psi^{\dagger} \psi,
$$

where $m$ is the current quark mass and $G$ is the four-quark effective coupling. The two-quark condensate is denoted as $\sigma_{1}$. The effective quark mass is defined as

$$
M=m-2 G \sigma_{1},
$$

with the two-quark condensate defined as

$$
\sigma_{1}=\langle\bar{\psi} \psi\rangle=-\int \frac{d^{4} p}{(2 \pi)^{4}} \operatorname{Tr}[S(p)]
$$

where $S(p)$ is the dressed quark propagator and the trace is taken in color, flavor, and Dirac spaces. The integration is divergent, and a cutoff $\Lambda$ on the momentum is usually used. In this case, the chemical potential $\mu$ must be less than the cutoff $\Lambda$ so as to get a reliable result, setting an upper limit for the weighting parameter $\alpha$ [22]. To circumvent this defect, we introduce the proper-time regularization here. The key equation is a replacement

$$
\frac{1}{A\left(p^{2}\right)^{n}} \rightarrow \frac{1}{(n-1) !} \int_{\tau_{\mathrm{UV}}}^{\infty} d \tau \tau^{n-1} e^{-\tau A\left(p^{2}\right)},
$$

where $\tau_{\mathrm{UV}}$ is introduced to regularize the ultraviolet divergence.

Beyond the chiral limit, three parameters $\left(\tau_{\mathrm{UV}}, G\right.$, and $\left.m\right)$ need to be fixed by requirements such as the two-quark condensate derived from QCD sum rules or lattice QCD, 
pion decay constant, or pion mass which are the results of chiral symmetry breaking. Since the Gell-Mann-OakesRenner relation is satisfied in low energy, it also can be used to calibrate these parameters. Fixing $m=5.0 \mathrm{MeV}$, with $G=3.086 \times 10^{-6} \mathrm{MeV}^{-2}$, and $\tau_{\mathrm{UV}}=1092 \mathrm{MeV}$, it gives $f_{\pi}=93 \mathrm{MeV}$ and $m_{\pi}=135 \mathrm{MeV}$. At a zero temperature, the condensate is

$$
\begin{aligned}
\langle\bar{\psi} \psi\rangle= & -2 N_{c} \sum_{u, d} \int \frac{d^{3} p}{(2 \pi)^{3}} \frac{M}{E_{p}}\left(1-\theta\left(\mu-E_{p}\right)\right) \\
= & -2 N_{c} \sum_{u, d}\left(\int \frac{d^{3} p}{(2 \pi)^{3}} \int_{\tau_{\mathrm{UV}}}^{\infty} d \tau \frac{e^{-\tau E^{2}}}{\sqrt{\pi \tau}}\right. \\
& \left.-\int \frac{d^{3} p}{(2 \pi)^{3}} \frac{M}{E_{p}} \theta\left(\mu-E_{p}\right)\right),
\end{aligned}
$$

with $E_{p}=\sqrt{\vec{p}^{2}+M^{2}}$.

As a purely technical device to examine the effect of a rearrangement of fermion field operators, the Fierz transformation of $L_{\mathrm{NJL}}$ is

$$
\begin{aligned}
\mathcal{L}_{\text {Fierz }}= & \bar{\psi}(i \not \gamma-m) \psi+\frac{G}{8 N_{c}}\left[2(\bar{\psi} \psi)^{2}+2\left(\bar{\psi} i \gamma^{5} \vec{\tau} \psi\right)^{2}\right. \\
& -2(\bar{\psi} \vec{\tau} \psi)^{2}-2\left(\bar{\psi} i \gamma^{5} \psi\right)^{2}-4\left(\bar{\psi} \gamma^{\mu} \psi\right)^{2} \\
& \left.-4\left(\bar{\psi} \gamma^{\mu} \gamma^{5} \psi\right)^{2}+\left(\bar{\psi} \sigma^{\mu \nu} \psi\right)^{2}-\left(\bar{\psi} \sigma^{\mu \nu} \vec{\tau} \psi\right)^{2}\right] .
\end{aligned}
$$

The mean-field-approximation result is

$$
\begin{aligned}
\mathcal{L}_{2}= & \bar{\psi}(i \not \partial-m) \psi+\frac{G}{2 N_{c}} \sigma_{1} \bar{\psi} \psi+G \sigma_{1}^{2} \\
& +\mu \psi^{\dagger} \psi-\frac{G}{N_{c}} \sigma_{2} \psi^{\dagger} \psi+\frac{G}{2 N_{c}} \sigma_{2}^{2},
\end{aligned}
$$

with $\sigma_{2}=\left\langle\psi^{\dagger} \psi\right\rangle$. Apparently, the effective quark mass and chemical potential in the Fierz-transformed Lagrangian can be defined, respectively, as

$$
\begin{gathered}
M=m-\frac{G}{2 N_{c}} \sigma_{1}, \\
\mu_{r}=\mu-\frac{G}{N_{c}} \sigma_{2} .
\end{gathered}
$$

Although the two formulas [Eqs. (3) and (9)] look different, in fact, when the parameters are recalibrated with different values of coupling $G$, they get the same result of the quark mass. If the NJL Lagrangian and its Fierz transformation are combined according to the literature [18], the second term in Eq. (9) is equivalent only to the next-toleading-order term of large $N_{c}$ expansion. The amending in the effective chemical potential almost can also be neglected.

However, the equivalence of $L_{\mathrm{NJL}}$ and $L_{\mathrm{Fierz}}$ means their linear combination with any complex $\alpha$, and in the meanfield approximation it reads

$$
\mathcal{L}_{C}=(1-\alpha) \mathcal{L}_{1}+\alpha \mathcal{L}_{2}
$$

With this combination, the effective mass and chemical potential are, respectively,

$$
\begin{gathered}
M=m-2\left(1-\alpha+\frac{\alpha}{4 N_{c}}\right) G \sigma_{1}, \\
\mu_{r}=\mu-\frac{\alpha G}{N_{c}} \sigma_{2} .
\end{gathered}
$$

Since the coefficient $\left(1-\alpha+\frac{\alpha}{4 N_{c}}\right) G$ in Eq. (12) requires fitting low-energy data, we can redefine the effective coupling as

$$
G^{\prime}=\left(1-\alpha+\frac{\alpha}{4 N_{c}}\right) G
$$

Thus, the quark mass and chemical potential are rewritten, respectively, as

$$
\begin{gathered}
M=m-2 G^{\prime} \sigma_{1}, \\
\mu_{r}=\mu-\frac{G^{\prime}}{N_{c}} \frac{\alpha}{1-\alpha+\frac{\alpha}{4 N_{c}}} \sigma_{2} .
\end{gathered}
$$

Only for $\alpha<1$, this is mathematically equivalent to adding a vector-isoscalar channel in the $L_{\mathrm{NJL}}$ [38]. By introducing a positive value of $\alpha$, one counts in more terms that may be ignored in the mean-field approximation with only $L_{\mathrm{NJL}}$. So, in this sense, the two approaches are different.

At a zero temperature, the quark number density is given by

$$
\rho_{u, d}=2 N_{c} \int \frac{d^{3} p}{(2 \pi)^{3}} \theta\left(\mu_{r}-E\right) .
$$

The $\alpha$ dependence of the effective quark mass and baryon number density are shown in Figs. 1 and 2, respectively. The quark mass decreases as the chemical potential increases. $\alpha$ has an impact on the effective quark mass. When $\alpha$ is greater than 0.9 , dependence of the quark mass on $\alpha$ is obvious. The quark baryon number density increases as the chemical potential increases. At a fixed chemical potential, the quark baryon number density decreases as $\alpha$ increases. Comparing Fig. 2, we can see that at the mean-field level the contribution of the effective potential from the Fierz-transformed Lagrangian to the baryon number density is negligible at a low chemical potential (for example, less than $400 \mathrm{MeV}$ ). Specifically, $\alpha=0$ is equivalent to not considering the contribution of the Fierz-transformed Lagrangian to the effective potential. That is, the contribution of the next-toleading-order term in large $N_{c}$ expansion is not considered, while the case of $\alpha \neq 0$ is equivalent to considering the influence of the next-to-leading-order term in large $N_{c}$ expansion on the effective potential. 


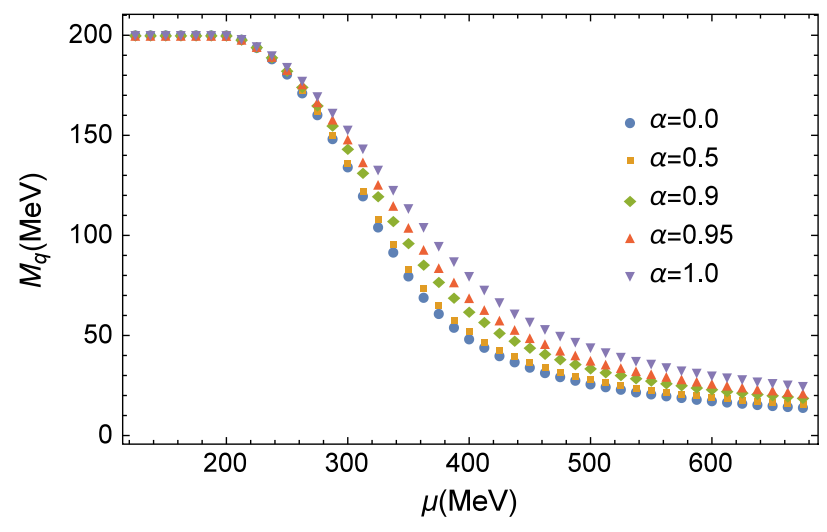

FIG. 1. The quark mass as a function of $\mu$ is presented. It is the standard NJL model result with $\alpha=0$ and the Fierz-transformed result with $\alpha=1$. With $\alpha=0.5$, it is the result of taking into account the next-to-leading order in large $N_{c}$ expansion, which has little impact on the effective quark mass.

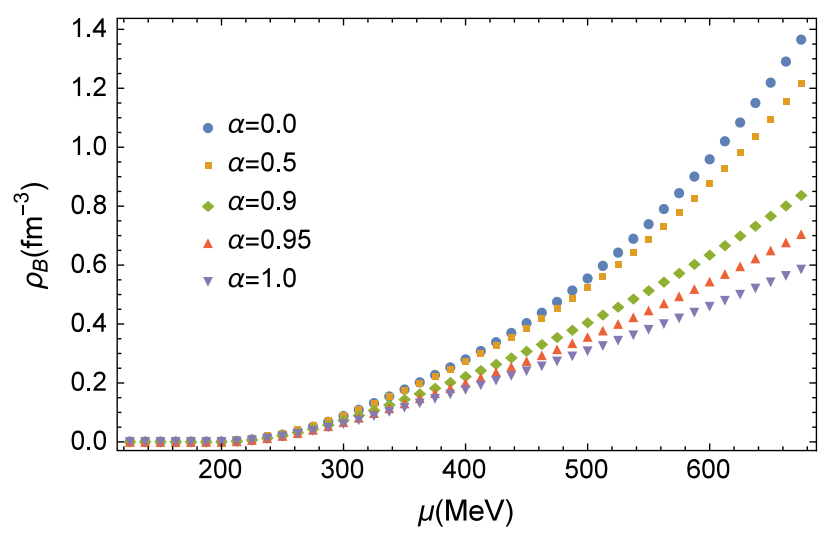

FIG. 2. The baryon number density as a function of $\mu$ is presented. When $\mu<M_{q}$, the quark number density $\rho(\mu)$ is zero. Also, $\rho(\mu)$ decreases as $\alpha$ increases and dramatically as $\alpha>0.5$ at large $\mu$.

\section{THE QUARK STAR STRUCTURES}

\section{A. The equation of state}

The EOS is the key to calculating the mass-radius relation and tidal formability $\Lambda$. The tidal formability measures the star's quadrupole deformation in response to the companion's perturbing tidal field during the merger of two stars.

The model-independent equations of state of strong interaction matter at finite $\mu$ and zero $T$ are $[39,40]$

$$
\begin{gathered}
P\left(\mu_{u}, \mu_{d}\right)=P(\mu=0)+\sum_{u, d} \int_{0}^{\mu} d \mu \rho(\mu), \\
\varepsilon\left(\mu_{u}, \mu_{d}\right)=-P\left(\mu_{u}, \mu_{d}\right)+\sum_{u, d} \mu \rho(\mu) .
\end{gathered}
$$

Here, $P(\mu=0)$ represents the vacuum pressure at $\mu=0$. In some works, $-P(\mu=0)$ is taken as a free parameter corresponding to the bag constant in the MIT bag model. In the standard NJL model, it is sometimes defined at a zero chemical potential as the pressure difference between results from the Nambu solution and bare quark propagator:

$$
P(\mu=0)=P\left(M_{N}\right)-P(m),
$$

where $M_{N}$ denotes the Nambu solution of the quark gap equation at $\mu=0$ and $m$ is the current quark mass. In this definition, the vacuum pressure is $P(\mu=0)=$ $-(129.71 \mathrm{MeV})^{4}$. However, $m$ is not a solution of the gap equation. A consistent definition is to take the difference between two solutions in analogy to the BCS theory. As suggested in Refs. $[26,30,31]$, the vacuum pressure in use is

$$
P(\mu=0)=P\left(M_{N}\right)-P\left(M_{W}\right),
$$

with $M_{W}$ the quasi-Wigner solution of the gap equation. In this case, the vacuum pressure is $P(\mu=0)=$ $-(131.75 \mathrm{MeV})^{4}$.

We have considered only pressure from quarks above. For a nonstrange quark star, we need the electron to keep electric charge neutrality:

$$
\frac{2}{3} \rho_{u}-\frac{1}{3} \rho_{d}-\rho_{e}=0
$$

with $\rho_{u}, \rho_{d}$, and $\rho_{e}$ being the number densities of the up and down quarks and electron, respectively. Then the pressure and energy density are

$$
\begin{gathered}
P_{\mathrm{tot}}=P\left(\mu_{u}, \mu_{d}\right)+\frac{\mu_{e}^{4}}{12 \pi^{2}}, \\
\varepsilon_{\mathrm{tot}}=\varepsilon\left(\mu_{u}, \mu_{d}\right)+\frac{\mu_{e}^{4}}{4 \pi^{2}},
\end{gathered}
$$

respectively. Here $\mu_{e}$ is the electron charge chemical potential, and the electron density is given by $\rho_{e}=$ $\mu_{e}^{3} /\left(3 \pi^{2}\right)$. We have to take into account the baryon number and electric charge conservation in weak decay $d \leftrightarrow$ $u+e+\bar{\nu}_{e}$. Then the chemical potential equilibrium gives $\mu_{d}=\mu_{u}+\mu_{e}$. Consequently, the EOS could be obtained. The EOSs with different $\alpha$ 's are plotted in Fig. 3. With a fixed negative pressure of the vacuum, the stiffness of EOS increases along with $\alpha$.

We are now ready to investigate the structure of a quark star using the Tolman-Oppenheimer-Volkoff equations (in units $G=c=1$ )

$$
\begin{gathered}
\frac{d P(r)}{d r}=-\frac{(\varepsilon+P)\left(M+4 \pi r^{3} P\right)}{r(r-2 M)}, \\
\frac{d M(r)}{d r}=4 \pi r^{2} \varepsilon
\end{gathered}
$$



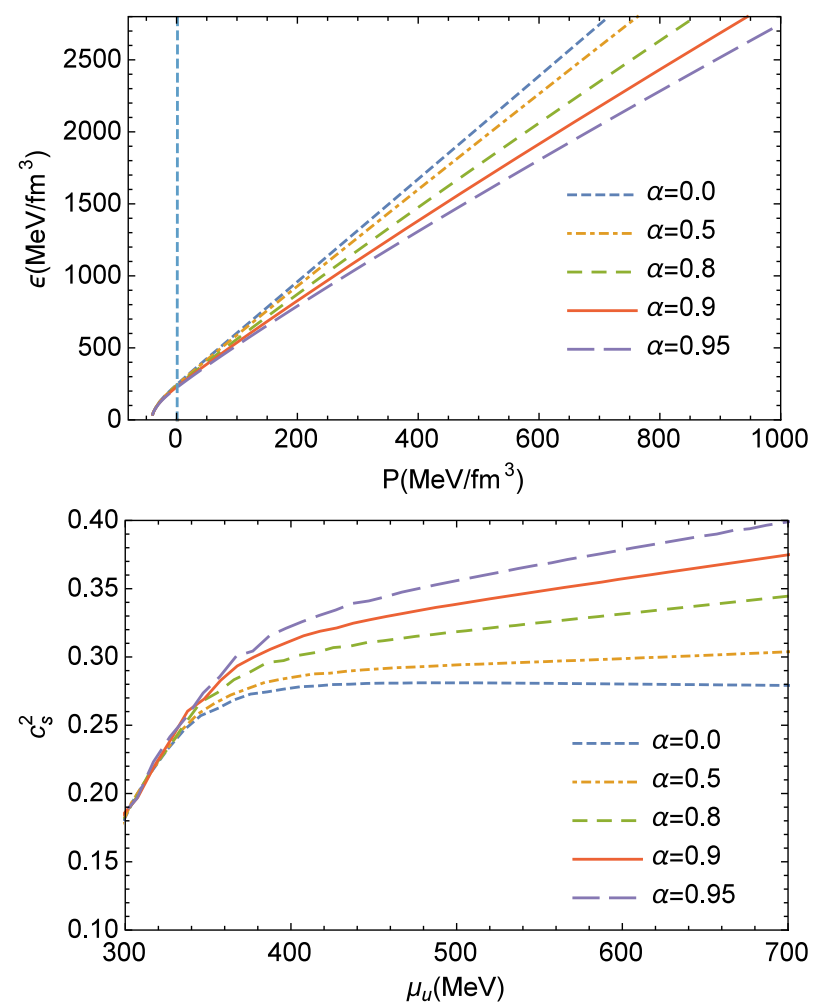

FIG. 3. The EOSs for different $\alpha$ 's are presented. The upper plot shows energy density $\varepsilon$ as a function of pressure $P$. The lower plot shows the square of the velocity of sound (defined as $\frac{d P}{d \varepsilon}$ ) as a function of $u$ quark chemical potential $\mu_{u}$. Zero energy density and negative pressure appear as $\mu_{u}<M_{q}$, and this part of the plot is not shown in the figure. Different $\alpha$ 's show different results only when $\mu_{u}$ is greater than $300 \mathrm{MeV}$. Both plots show that the EOS for smaller $\alpha$ becomes softer than the one for larger $\alpha$.

which give the mass-radius relation. We have calculated quark stars with Eq. (11) with different parameters $\alpha$. The mass-radius relation is presented in Fig. 4.

We first notice that, as $\alpha>0.9$, the maximum mass (denoted as $M_{\mathrm{TOV}}$ ) can be larger than 2.0 solar masses. This matches the observed pulsars mass of PSR J1614-2230 $\left(M=1.928 \pm 0.017 M_{\odot}\right)[3]$ and PSR J0348+0432 $\left(M=2.01 \pm 0.04 M_{\odot}\right)[4]$. We have also listed the surface energy densities $\varepsilon_{0}$ in Table I which satisfy the constraint that $\varepsilon_{0}$ should be larger than $2.80 \times 10^{14} \mathrm{~g} / \mathrm{cm}^{3}$. Second, the upper limit on the radius of a 1.4-solar-mass star from three recent works are $R \leq 13.76 \mathrm{~km}, R \leq 13.6 \mathrm{~km}$, and $8.9 \mathrm{~km} \leq R \leq 13.76 \mathrm{~km}$, respectively [41-43]. We see in Fig. 4 that the upper limits of the radius are all larger than our maximum radii and, thus, the radii of a 1.4-solar-mass star. The lower limit of the radius from Ref. [37] on a 1.6-solar-mass neutron star is $10.7 \mathrm{~km}$. The radii from our parameters $\alpha \geq 0.5$ satisfy this constraint. If the compact objects are quark stars, then in studying QCD matter at a high density, the Lagrangian without a Fierz transformation $(\alpha=0)$ is incomplete at the level of the mean-field

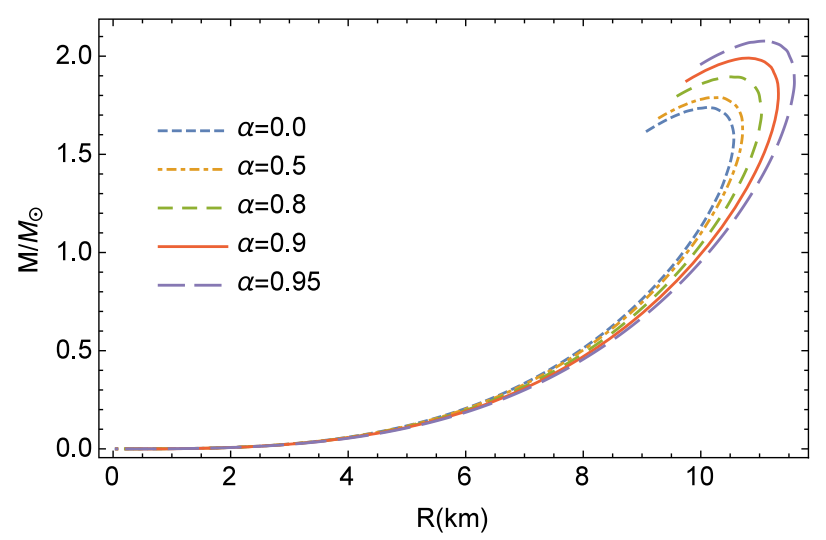

FIG. 4. The mass-radius relation of a quark star is presented. The maximum mass increases with $\alpha$. The maximum radii are less than $12 \mathrm{~km}$. When $\alpha=0.9$, the maximum mass is close to $2 M_{\odot}$ with a star radius of $10.87 \mathrm{~km}$. When $\alpha=0.95$, the maximum mass is larger than $2.05 M_{\odot}$. Only for the case $\alpha \geq 0.5$ are the radii of stars with a mass of $1.6 M_{\odot}$ larger than $10.7 \mathrm{~km}$.

approximation, while Eq. (11) provides a more realistic description.

\section{B. The tidal deformability}

During the merger of two stars, it is reasonable to think about the magnetic breaking during the merger evolution. The Love number measures the distortion of the shape of the surface of a star by an external tidal field. The tidal deformability is related to the $l=2$ dimensionless tidal Love number $k_{2}$. In units $G=c=1$, it is

$$
k_{2}=\frac{3}{2} \Lambda\left(\frac{M}{R}\right)^{5}
$$

The most recent analysis on the binary neutron star merger GW170817 has found tighter constraints on the component mass to lie between 1.00 and $1.89 M_{\odot}$ with $\Lambda$ in $(0,630)$ when allowing for large component spins and on the component masses to lie between 1.16 and $1.60 M_{\odot}$ with $\Lambda=$ $300_{-230}^{+420}$ when the spins are restricted to be within the range observed in Galactic binary neutron stars [32]. The early restriction on the tidal deformability $\Lambda$ for $1.4 M_{\odot}$ is less than 800 (1400) for the low- (high-) spin prior case [33,44].

TABLE I. Properties of a $u d$ quark star, including the maximum mass $M_{\mathrm{TOV}}$, the corresponding radius $R$, and surface energy density $\varepsilon_{0}$. The radii of a 1.6-solar-mass star are also listed. It is obvious that the maximum mass increases as $\alpha$ increases.

\begin{tabular}{lcccc}
\hline \hline$\alpha$ & $M_{\mathrm{TOV}}\left(M_{\odot}\right)$ & $R(\mathrm{~km})$ & $R_{1.6}(\mathrm{~km})$ & $\varepsilon_{0}\left(10^{14} \mathrm{~g} / \mathrm{cm}^{3}\right)$ \\
\hline 0.00 & 1.7376 & 10.17 & 10.56 & 4.3252 \\
0.50 & 1.7896 & 10.33 & 10.71 & 4.2647 \\
0.80 & 1.8949 & 10.50 & 10.98 & 4.1532 \\
0.90 & 1.9903 & 10.87 & 11.19 & 4.0412 \\
0.95 & 2.0773 & 11.14 & 11.37 & 3.9416 \\
\hline \hline
\end{tabular}


TABLE II. Properties of our 1.4-solar-mass quark star, including the compactness $M / R$, the Love number $k_{2}$, and the tidal deformability $\Lambda$. Since the radius increases as $\alpha$ increases, the compactness decreases as $\alpha$ increases. And the tidal deformability increases as $\alpha$ increases.

\begin{tabular}{lccc}
\hline \hline$\alpha$ & $M / R$ & $k_{2}$ & $\Lambda$ \\
\hline 0.00 & 0.1980 & 0.1435 & 314.26 \\
0.50 & 0.1962 & 0.1481 & 339.93 \\
0.80 & 0.1925 & 0.1570 & 395.06 \\
0.90 & 0.1895 & 0.1645 & 448.98 \\
0.95 & 0.1869 & 0.1708 & 499.37 \\
\hline \hline
\end{tabular}

In matching the interior and exterior solutions across the star surface, the $l=2$ tidal Love number $k_{2}$ for the internal solution is given by [45]

$$
\begin{aligned}
k_{2}= & \frac{8}{5} C^{5}(1-2 C)^{2}[2+2 C(y-1)-y] \\
& \times\{2 C[6-3 y+3 C(5 y-8)] \\
& +4 C^{3}\left[13-11 y+C(3 y-2)+2 C^{2}(1+y)\right] \\
& \left.+3(1-2 C)^{2}[2-y+2 C(y-1)] \ln (1-2 C)\right\}^{-1},
\end{aligned}
$$

where $C=M / R$ defines the compactness of the star and $y$ is related to the metric variable $H$ and surface energy density $\varepsilon_{0}$ :

$$
y=\frac{R \beta(R)}{H(R)}-\frac{4 \pi R^{3} \varepsilon_{0}}{M} .
$$

For some neutron star model, the surface energy density is zero. But in our NJL model with a negative vacuum pressure, the surface energy $\varepsilon_{0}$ is nonzero as shown in Fig. 3.

The metric variable $H$ related to the EOS can be obtained by integrating two differential equations:

$$
\begin{aligned}
\frac{d H(r)}{d r}= & \beta, \\
\frac{d \beta(r)}{d r}= & 2 g H\{-2 \pi[5 \varepsilon+9 P+f(\varepsilon+P)] \\
& \left.+\frac{3}{r^{2}}+2 g\left(\frac{M}{r^{2}}+4 \pi r P\right)^{2}\right\} \\
& +2 g \frac{\beta}{r}\left[-1+\frac{M}{r}+2 \pi r^{2}(\varepsilon-P)\right],
\end{aligned}
$$

where $g=(1-2 M / r)^{-1}$ and $f=d \varepsilon / d P$. The iteration starts from the center at $r=0$ via expansions $H(r)=a_{0} r^{2}$ and $\beta(r)=2 a_{0} r$ with constant $a_{0}$. As can be seen from Eq. (29), we concern only the ratio $\beta / H$. So $a_{0}$ can be arbitrarily chosen in a numerical calculation. The Love number $k_{2}$ and tidal deformability $\Lambda$ for different $\alpha$ 's are calculated and presented in Table II. They increase as $\alpha$ increases. For a $1.4 M_{\odot}$ star, all the calculated tidal deformabilities lie within a reasonable range, i.e., less than the upper limit of a low-spin star and large than the lower limits $\Lambda_{1.4}>200$ [37].

\section{SUMMARY}

Using a recently proposed generalized NJL model [Eq. (11)] with a new parameter $\alpha$ incorporating different interaction channels [20], we studied the structure of light quark stars in this work. We find the EOS of a star gets harder with a larger $\alpha$, namely, with more contribution from the Fierz-transformed term $L_{\text {Fierz }}$. We use proper-time regularization to treat the ultradivergence, so there is no upper limit for the choice of $\alpha$. This improves upon the momentum cutoff regularization scheme used in Ref. [22]. The weighting parameter $\alpha$ is the only free parameter. Other than to set the vacuum pressure to be a free parameter or to define the vacuum pressure with a current quark as in Eq. (20), our vacuum pressure is fixed to be the difference between the pressures from the Nambu solution and the quasi-Wigner solution [30]. Then the corresponding bag constant is obtained to be $B^{1 / 4}=131.75 \mathrm{MeV}$.

From the TOV equations, the mass-radius relation and tidal deformability are calculated for different $\alpha$ 's. As $\alpha>0.9$, the 2.0 solar mass can be yielded, which matches the masses of PSR J1614-2230 $\left(M=1.928 \pm 0.017 M_{\odot}\right)$ and PSR J0348+0432 $\left(M=2.01 \pm 0.04 M_{\odot}\right)$. Our results of the surface energy density are larger than $2.80 \times 10^{14} \mathrm{~g} / \mathrm{cm}^{3}$, and the radii for a 1.4-solar-mass star satisfy the constraints $R \leq 13.76 \mathrm{~km}$ and $R \leq 13.6 \mathrm{~km}$ [41,42]. The lower limit $10.7 \mathrm{~km}$ of a 1.6-solar-mass neutron star is satisfied for $\alpha \geq 0.5$. This suggests that the Fierztransformed Lagrangian must be included in the combined Lagrangian. We have also calculated the tidal Love number $k_{2}$ and the tidal deformability $\Lambda$. The tidal deformability $\Lambda$ calculated for a 1.4-solar-mass star increases with $\alpha$, is within the interval $(200<\Lambda<800)$ [37] and the interval $(0<\Lambda<630)$ for large component spin, and satisfies the constraint with $\Lambda=300_{-230}^{+420}$ when restricting the magnitude of the component spins from the analysis of GW170817 [32]. Admitting the nonstrange quark, our improved NJL model therefore provides a consistent explanation to a variety of astronomical observations.

\section{ACKNOWLEDGMENTS}

This work is supported in part by the National Natural Science Foundation of China (under Grants No. 11475085, No. 11535005, No. 11690030 , No. 11873030, and No. 11905104) and the National Major state Basic Research and Development of China (Grant No. 2016YFE0129300). 
[1] E. Witten, Phys. Rev. D 30, 272 (1984).

[2] P. Demorest, T. Pennucci, S. M. Ransom, M. S. E. Roberts, and J. W. T. Hessels, Nature (London) 467, 1081 (2010).

[3] E. Fonseca, T. T. Pennucci, J. A. Ellis, I. H. Stairs, D. J. Nice, S. M. nsom, P. B. Demorest, Z. Arzoumanian, K. Crowter, T. Dolch, R. D. Ferdman, M. Gonzalez, G. Jones, M. L. Jones, M. T. Lam, L. Levin, M. A. McLaughlin, K. ovall, J. K. Swiggum, and W. Zhu, Astrophys. J. 832, 167 (2016).

[4] J. Antoniadis et al., Science 340, 1233232 (2013).

[5] H. T. Cromartie et al., Nat. Astron., 10.1038/s41550-0190880-2 (2019).

[6] A. Dobado, F. J. Llanes-Estrada, and J. A. Oller, Phys. Rev. C 85, 012801 (2012).

[7] E. Babichev and D. Langlois, Phys. Rev. D 81, 124051 (2010).

[8] S. Ray, A. L. Espindola, M. Malheiro, J. P. S. Lemos, and V. T. Zanchin Phys. Rev. D 68, 084004 (2003).

[9] C. R. Ghezzi, Phys. Rev. D 72, 104017 (2005).

[10] R. P. Negreiros, F. Weber, M. Malheiro, and V. Usov, Phys. Rev. D 80, 083006 (2009).

[11] G. B. Cook, S. L. Shapirol, and S. A. Teukolsky, Astrophys. J. 424, 823 (1994).

[12] N. Stergioulas and J. L. Friedman, Astrophys. J. 444, 306 (1995).

[13] P. G. Krastev, B. A. Li, and A. Worley, Astrophys. J. 676, 1170 (2008).

[14] B. Holdom, J. Ren, and C.Zhang, Phys. Rev. Lett. 120, 222001 (2018).

[15] A. R. Bodmer, Phys. Rev. D 4, 1601 (1971).

[16] H. Terazawa, J. Phys. Soc. Jpn. 58, 3555 (1989); 58, 4388 (1989); 59, 1199 (1990).

[17] M. Buballa, Phys. Rep. 407, 205 (2005).

[18] S. P. Klevansky, Rev. Mod. Phys. 64, 649 (1992).

[19] T. Kunihiro and R. Hatsuda, Prog. Theor. Phys. 74, 765 (1985).

[20] F. Wang, Y. Cao, and H. Zong, Chin. Phys. C 43, 084102 (2019).

[21] Q. Y. Wang, T. Zhao, and H. S. Zong, arXiv:1908.01325.

[22] T. Zhao, W. Zheng, F. Wang, C. M. Li, Y. Yan, Y. F. Huang, and H. S. Zong, Phys. Rev. D 100, 043018 (2019).

[23] Y. Yan, J. Cao, X. L. Luo, W. M. Sun, and H. Zong, Phys. Rev. D 86, 114028 (2012).

[24] A. Chodos, R. L. Jaffe, K. Johnson, C. B. Thorn, and V. F. Weisskopf, Phys. Rev. D 9, 3471 (1974).
[25] C. Alcock, E. Farhi, and A. Olinto, Astrophys. J. 310, 261 (1986).

[26] C.-M. Li, P.-L. Yin, and H.-S. Zong, Phys. Rev. D 99, 076006 (2019).

[27] M. Alford, M. Braby, M. Paris, and S. Reddy, Astrophys. J. 629, 969 (2005).

[28] E.-P. Zhou, X. Zhou, and A. Li, Phys. Rev. D 97, 083015 (2018).

[29] B.-L. Li, Z.-F. Cui, Z.-H. Yu, Y. Yan, S. An, and H.-S. Zong, Phys. Rev. D 99, 043001 (2019).

[30] S.-S. Xu, Z.-F. Cui, A. Sun, and H.-S. Zong, J. Phys. G 45, 105001 (2018).

[31] Z.-F. Cui, S.-S. Xu, B.-L. Li, A. Sun, J.-B. Zhang, and H.-S. Zong, Eur. Phys. J. C 78, 770 (2018).

[32] B. P. Abbott, R. Abbott, T. D. Abbott, F. Acernese, K. Ackley, C. Adams, T. Adams, P. Addesso et al. (LIGO Scientific and Virgo Collaboration), Phys. Rev. X 9, 011001 (2019).

[33] B. P. Abbott, R. Abbott, T. D. Abbott, F. Acernese, K. Ackley, C. Adams, T. Adams, P. Addesso et al. (LIGO Scientific and Virgo Collaborations), Phys. Rev. Lett. 119, 161101 (2017).

[34] H. Gao, Z. Cao, S. Ai, and B. Zhang, Astrophys. J. Lett. 851, L45 (2017).

[35] A. Bauswein, O. Just, H.-T. Janka, and N. Stergioulas, Astrophys. J. Lett. 850, L34 (2017).

[36] J. E. Christian, A. Zacchi, and J. Schaffner-Bielich, Phys. Rev. D 99, 023009 (2019).

[37] A. Bauswein, AIP Conf. Proc. 2127, 020013 (2019).

[38] S. Benic, D. Blaschke, D. E. Alvarex-Castillo, T. Fisher, and S. Typel, Astron. Astrophys. 577, A40 (2015).

[39] H.-S. Zong and W.-M. Sun, Phys. Rev. D 78, 054001 (2008).

[40] H.-S. Zong and W.-M. Sun, Int. J. Mod. Phys. A 23, 3591 (2008).

[41] F. J. Fattoyev, J. Piekarewicz, and C. J. Horowitz, Phys. Rev. Lett. 120, 172702 (2018).

[42] E. Annala, T. Gorda, A. Kurkela, and A. Vuorinen, Phys. Rev. Lett. 120, 172703 (2018).

[43] S. De, D. Finstad, J. M. Lattimer, D. A. Brown, E. Berger, and C. M. Biwer, Phys. Rev. Lett. 121, 091102 (2018).

[44] B. Margalit and B. D. Metzger, Astrophys. J. Lett. 850, L19 (2017).

[45] T. Damour and A. Nagar, Phys. Rev. D 80, 084035 (2009). 